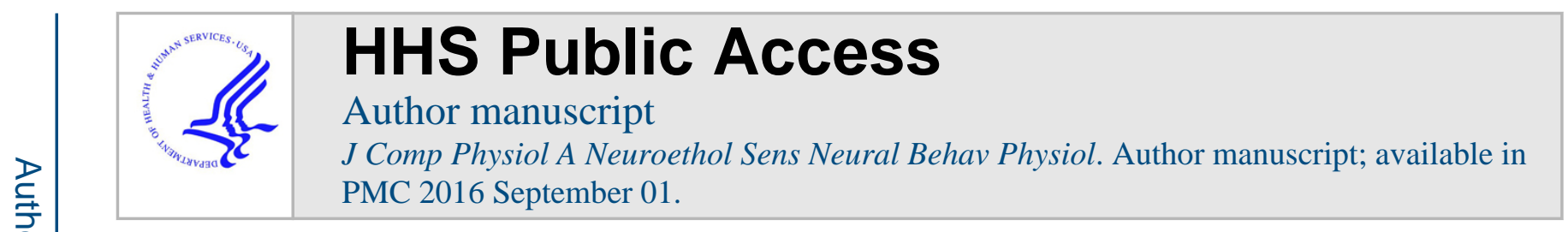

Published in final edited form as:

J Comp Physiol A Neuroethol Sens Neural Behav Physiol. 2015 September ; 201(9): 857-867. doi:

10.1007/s00359-015-1021-8.

\title{
Thermosensation and longevity
}

\author{
Rui Xiao ${ }^{1}$, Jianfeng $\mathrm{Liu}^{2}$, and X.Z. Shawn $\mathrm{Xu}^{1}$ \\ ${ }^{1}$ Life Sciences Institute and Department of Molecular \& Integrative Physiology, University of \\ Michigan, 210 Washtenaw Avenue, Ann Arbor, Michigan 48109, USA \\ ${ }^{2}$ College of Life Science and Technology, Collaborative Innovation Center for Brain Science, and \\ Key Laboratory of Molecular Biophysics of MOE, Huazhong University of Science and \\ Technology, Wuhan, Hubei 430074, China
}

\begin{abstract}
Temperature has profound effects on behavior and aging in both poikilotherms and homeotherms. To thrive under the ever fluctuating environmental temperatures, animals have evolved sophisticated mechanisms to sense and adapt to temperature changes. Animals sense temperature through various molecular thermosensors, such as thermosensitive TRP channels expressed in neurons, keratinocytes, and intestine. These evolutionarily conserved thermosensitive TRP channels feature distinct activation thresholds, thereby covering a wide spectrum of ambient temperature. Temperature changes trigger complex thermosensory behaviors. Due to the simplicity of the nervous system in model organisms such as C. elegans and Drosophila, the mechanisms of thermosensory behaviors in these species have been extensively studied at the circuit and molecular levels. While much is known about temperature regulation of behavior, it remains largely unclear how temperature affects aging. Recent studies in C. elegans demonstrate that temperature modulation of longevity is not simply a passive thermodynamic phenomenon as suggested by the rate-of-living theory, but rather a process that is actively regulated by genes, including those encoding thermosensitive TRP channels. In this review, we discuss our current understanding of thermosensation and its role in aging.
\end{abstract}

\section{Keywords}

Thermal; Thermotaxis; Lifespan; Heat; Cold

\section{Introduction}

Both genetic and environmental factors affect aging. In the past two decades, studies from model organisms such as worms, flies, and mice have identified a number of evolutionarily conserved genetic pathways, such as insulin and insulin-like growth factor 1 (IGF1) signaling (IIS), that modulate aging (Finch and Ruvkun 2001; Kenyon 2010). On the other hand, relatively less is known about the mechanisms by which environmental factors

Corresponding author's address X.Z. Shawn Xu, Ph.D., Life Sciences Institute, University of Michigan, 210 Washtenaw Avenue, Ann Arbor, Michigan 48109, USA. Tel.: 734-615-9311; shawnxu @umich.edu. 
regulate longevity, although studies have suggested that the environment is an equally, if not more, important determinant (up to $80 \%$ ) of aging in many organisms, including humans (Gurland et al. 2004; Herskind et al. 1996).

Food and temperature are the two primary environmental factors that affect lifespan in nearly all tested animal models. Nutrient, odor and tastant in the food all regulate longevity, and dietary restriction (DR) is considered one of the most effective means to extend lifespan (Gems and Partridge 2013; Kenyon 2010; Mair and Dillin 2008). Food regulates lifespan through various diet-sensitive genes and signaling pathways. Recent work has identified a number of genetic pathways (e.g. mTOR signaling, insulin signaling, AMPK, autophagy signaling, etc) through which DR extends lifespan (Bishop and Guarente 2007; Mair and Dillin 2008). By contrast, very little is known about how temperature affects longevity.

Temperature modulates a plethora of physiological and behavioral processes such as aging, development, reproduction, mating, and circadian rhythm (Cabanac 1975; Clarke and Portner 2010). With the exception of extreme temperatures which may threaten survival, poikilothermic animals are long known to live longer at lower environmental temperatures, and vice versa. This phenomenon was first documented a century ago (Loeb 1908; Loeb and Northrop 1916). Interestingly, lower core body temperature was also shown to extend lifespan in homeothermic mice (Conti et al. 2006), implying a general role of temperature in lifespan modulation. Historically, the rate-of-living theory was adopted to explain the apparent inverse correlation between longevity and ambient temperatures (Lints 1989). According to this theory, lower temperatures reduce the rate of chemical reactions of living species, thereby slowing down the pace of aging. This theory suggests that temperatureregulated longevity is purely a passive thermodynamic phenomenon. However, since genes have been shown to actively mediate the effect of food on longevity, one may question why temperature would be an exception (Finch and Ruvkun 2001; Kenyon 2010).

Animals detect temperature changes through their sensory systems (McKemy 2007). Temperature modulates both behavior and aging. Strikingly, animals across species have evolved evolutionarily conserved mechanisms to sense temperature. Namely, distinct types of temperature-sensitive membrane proteins (ion channels and membrane receptors) are widely involved in thermosensation in all metazoans (Dhaka et al. 2006; Jordt et al. 2003). In this review, we will briefly discuss our current understanding of thermosensation and its potential roles in behavior and aging, with a focus on aging.

\section{Molecular sensors of temperature}

Among various temperature-sensitive membrane proteins, a subset of thermosensitive transient receptor potential (TRP) channels (called ThermoTRPs) has been extensively characterized in the past two decades. TRP channels were first cloned in Drosophila and later found to form a family of evolutionarily conserved non-selective cation channels (Montell and Rubin 1989; Venkatachalam and Montell 2007). As cellular sensors, TRP channels play important roles in chemosensation, mechanosensation, osmosensation, photosensation, and thermosensation in diverse organisms (Venkatachalam and Montell 
2007; Xiao and Xu 2009). These channels are found in C. elegans (17 members), Drosophila (13 members), and mammals (28 members), and together they form 7 subfamilies, including TRPC (TRP-Canonical), TRPV (TRP-Vanilloid), TRPM (TRPMelastatin), TRPN (TRP-NompC), TRPA (TRP-Ankyrin), TRPP (TRP-Polycystin), and TRPML (TRP-MucoLipin) (Fowler and Montell 2013; Venkatachalam and Montell 2007; Xiao and Xu 2009).

ThermoTRPs, a subset of TRP proteins found from at least 4 TRP subfamilies (TRPV, TRPA, TRPM, and TRPP), display superb temperature sensitivity and play critical roles in thermosensation and other temperature-regulated processes in a wide spectrum of organisms ranging from C. elegans to humans. ThermoTRPs are typically featured with remarkably high $\mathrm{Q}_{10}$ coefficient values (a measure of the reaction rate change as a function of temperature increase by $10^{\circ} \mathrm{C}$ ). For example, the heat and capsaicin receptor TRPV1 has a $\mathrm{Q}_{10}$ value $>20$, whereas most temperature-insensitive enzymatic reactions and ion channel gating exhibit a $Q_{10}$ value <3 (Clapham and Miller 2011; Hille 2001). Functional studies using electrophysiology and calcium imaging have identified multiple thermoTRPs and other types of temperature-sensitive ion channels and receptors such as two-pore domain potassium channels (KCNKs), rhodopsin, and certain insect gustatory receptors (Dhaka et al. 2006; Jordt et al. 2003; Lotshaw 2007; Luo et al. 2011; Ni et al. 2013; Shen et al. 2011). In this review, we will mainly focus on thermoTRPs as they are the ones that have been best characterized.

\section{A) Mammalian thermosensors}

Mammalian peripheral thermosensation is mainly mediated by sensory neurons located in the dorsal root ganglia (DRG) and trigeminal ganglia (TG) (Vriens et al. 2014).

Additionally, skin keratinocytes play a role in thermosensation by signaling to epidermal nerve endings (Caterina 2014).

Investigators have identified at least 7 thermoTRPs from thermosensitive tissues in mammals, including TRPM8 and TRPA1 which are cold-sensitive, and TRPV1-TRPV4 and TRPM3 which are heat-sensitive (Ramsey et al. 2006; Venkatachalam and Montell 2007) (Fig. 1a). These thermoTRPs are widely expressed in various temperature-sensing tissues as described above and have distinct temperature activation thresholds, thereby covering a wide spectrum of ambient temperature. Interestingly, the temperature sensitivity of some thermoTRPs appears to be species specific. For example, TRPA1 is a cold-sensitive channel in C. elegans and mice but functions as a heat-sensitive channel in flies and snakes (Chatzigeorgiou et al. 2010; Gracheva et al. 2010; Story et al. 2003; Viswanath et al. 2003; Xiao et al. 2013). Similarly, mammalian TRPV3 is activated by warming, yet amphibian TRPV3 is a cold-sensitive channel (Saito et al. 2011). In addition, different isoforms of certain thermoTRPs can exhibit distinct temperature activation thresholds. For instance, a trigeminal ganglia-specific splicing isoform of TRPV1 from vampire bats features a thermal activation threshold of $\sim 30^{\circ} \mathrm{C}$ which is significantly lower than other TRPV1 isoforms, endowing vampires bats with an exceptional thermosensitivity (Gracheva et al. 2011). A similar phenomenon is also present among distinct isoforms of Drosophila TRPA1 (Kang et 
al. 2012; Zhong et al. 2012). The temperature sensitivity of distinct thermoTRPs and other thermosensitive ion channels may be largely determined by the heat capacity during channel gating, and thus altering a few residues may switch the directionality of thermosensitivity or even confer thermosensitivity to an otherwise thermo-insensitive channel (Chowdhury et al. 2014; Jabba et al. 2014; Yang and Zheng 2014). It should be noted that knockout mice of thermoTRPs still exhibit considerable temperature sensitivity, implying that unknown thermosensors must be present in mammals and other species.

\section{B) Drosophila thermosensors}

Drosophila larvae and adults are highly sensitive to changes in environmental temperatures. At the cellular level, class IV multidendritic (MD) neurons in the periphery sense hot temperatures $\left(>39^{\circ} \mathrm{C}\right.$ ) (Tracey et al. 2003), while anterior cell (AC) neurons in the brain mediate warmth sensing $\left(>24^{\circ} \mathrm{C}\right.$ ) (Hamada et al. 2008). In addition, distinct groups of antennal neurons in the periphery sense cool and warm temperatures (Gallio et al. 2011; Kwon et al. 2010; Rosenzweig et al. 2008). Other less characterized temperature-sensing organs and cells are also present in flies (Fowler and Montell 2013).

Genetic analysis and behavioral assays are widely used to identify and characterize thermoTRPs in Drosophila. At least 3 TRP channels (TRPA1, Painless, and Pyrexia) are heat-sensitive, all of which belong to the TRPA subfamily (Lee et al. 2005; Lee and Montell 2013; Tracey et al. 2003; Viswanath et al. 2003) (Fig. 1b). These TRPA channels may be directly activated by heat. Additionally, thermotaxis behavioral assays suggest that the TRPC channel TRPL, the TRPV channel Inactive, and the TRPP1 homologs Brivido1-3 play important roles in cold sensation (Gallio et al. 2011; Kwon et al. 2010; Rosenzweig et al. 2008) (Fig. 1b). However, none of these channels has been demonstrated to be directly activated by cooling in vitro. It remains possible that they may function as transduction channels in cold sensation. The molecular identities of direct cold sensors in flies require further investigation.

\section{C) C. elegans thermosensors}

C. elegans is also highly sensitive to temperature changes. AFD neuron is the best studied heat-sensitive neuron in C. elegans (Garrity et al. 2010; Mori et al. 2007). In addition, AWC, ASI, FLP and PHC neurons may also play a role in sensing heat (Chatzigeorgiou et al. 2010; Liu et al. 2012; Mohammadi et al. 2013; Mori et al. 2007). Interestingly, PVD neuron can respond to both heat and cold in C. elegans (Chatzigeorgiou et al. 2010; Mohammadi et al. 2013; Xiao et al. 2013). While neurons dedicated to cold sensing have not been identified in C. elegans, a recent study has shown that C. elegans intestine is highly sensitive to cooling, expanding the repertoire of cold receptors from neurons to non-excitable cells (Xiao et al. 2013). As C. elegans intestine is in fact its fat tissue (McGhee 2007), this study suggests that fat tissues in other organisms may also be sensitive to cooling (Xiao et al. 2013). Indeed, a recent study reported that mammalian fat cells show temperature sensitivity (Ye et al. 2013).

In $C$. elegans, TRPA-1 can be directly activated by temperature drop $\left(<20{ }^{\circ} \mathrm{C}\right)$ in both the intestine and PVD neuron (Chatzigeorgiou et al. 2010; Xiao et al. 2013) (Fig. 1c), 
supporting their function as a cold receptor. On the other hand, although the cyclic nucleotide-gated (CNG) channel TAX-2/TAX-4 can be activated by warming and closed by cooling in AFD neuron (Ramot et al. 2008), this type of channels require intracellular signaling for activation and are probably not temperature-gated per se (Ramot et al. 2008). The molecular identity of the heat sensor(s) in AFD neuron remains to be identified. In addition, the TRPV channels OSM-9 and OCR-2 seem to be involved in heat avoidance behavior (Chatzigeorgiou and Schafer 2011; Glauser et al. 2011; Liu et al. 2012; Mohammadi et al. 2013). For example, OSM-9 and OCR-2 may play a role in sensing noxious heat $\left(>35^{\circ} \mathrm{C}\right)$ in FLP neuron in the head and PHC neuron in the tail (Chatzigeorgiou and Schafer 2011; Liu et al. 2012), while OCR-2, but not OSM-9, is required for this response mediated by PVD neuron at the midbody (Mohammadi et al. 2013). However, the temperature sensitivity of these C. elegans TRPV channels was not fully characterized. As these channels can also be activated by $\mathrm{G}$ protein signaling (KahnKirby and Bargmann 2006; Xiao and Xu 2009), it is not clear whether they act as thermosensors or transduction channels.

\section{Temperature and behaviors}

Temperature changes drive complex behavioral responses in diverse organisms. Due to the simplicity of the nervous systems of simple model organisms such as C. elegans and Drosophila, it is feasible to map the neural circuits underlying thermosensory behaviors in these organisms.

The nervous system of adult $C$. elegans hermaphrodites contains only 302 neurons. As a result, the complete wiring diagram has been reconstituted by electron microscopy (White et al. 1986). Though equipped with a simple nervous system, C. elegans is capable of most sensations, including photosensation, chemosensation, touch sensation, proprioception, osmosensation, and thermosensation (Bargmann 2006; Bounoutas and Chalfie 2007; Garrity et al. 2010; Li et al. 2006; Ward et al. 2008; Xiao and Xu 2009). In the case of thermosensation, C. elegans remembers its past cultivation temperature and tends to move towards that temperature on a temperature gradient, a phenomenon termed thermotaxis (Hedgecock and Russell 1975). The neural and genetic mechanisms underlying thermotaxis have been well studied and extensively reviewed elsewhere (Fig. 2a) (Garrity et al. 2010; Luo et al. 2014; Mori et al. 2007). Briefly, AFD appears to be the principle thermosensory neuron mediating thermotaxis behavior, though other sensory neurons (e.g. AWC and ASI) are also involved (Fig. 2a). AFD, together with the interneurons AIY, AIZ and RIA, forms the core of the neural circuitry underlying thermotaxis (Fig. 2a). AFD senses temperature through an unknown thermosensor which transduces signals through cGMP signaling, leading to opening of the CNG channel TAX-2/TAX-4. In addition to thermotaxis, $C$. elegans exhibits robust escape responses to noxious heat stimuli (Glauser et al. 2011; Liu et al. 2012; Mohammadi et al. 2013; Wittenburg and Baumeister 1999). Neuropeptide signaling and the TRPV channels OSM-9 and OCR-2 may regulate the threshold of noxious heat avoidance behavior in C. elegans (Glauser et al. 2011; Liu et al. 2012; Mohammadi et al. 2013). Furthermore, the nociceptor PVD neuron seems to be involved in thermal avoidance behavior by sensing noxious heat at the midbody (Mohammadi et al. 2013). 
ThermoTRPs also play important roles in fly thermosensory behaviors. For example, TRPA1 is required in a small number of central brain neurons for Drosophila thermotaxis behavior, in which case it acts as a transduction channel downstream of $\mathrm{G}$ protein and phospholipase C (PLC) signaling (Kwon et al. 2008; Rosenzweig et al. 2005). Rhodopsin is required for this behavior likely as a direct thermosensor (Shen et al. 2011). The other two fly TRPA channels, Painless and Pyrexia, have higher temperature activation thresholds and are important for mediating noxious heat avoidance behavior (Lee et al. 2005; Tracey et al. 2003). In flies, peripheral thermosensory inputs converge onto three regions in the brain: mushroom body, lateral horn, and posterior lateral protocerebrum through projection neurons (Frank et al. 2015; Liu et al. 2015). These projection neurons can be excited by warming, cooling, or both (Frank et al. 2015; Liu et al. 2015). Heat-sensitive projection neurons receive excitatory inputs from heat receptors, as well as crossover inhibitory inputs from cold receptors via inhibitory interneurons. Through this mechanism, non-thermal noises could be cancelled while thermal activities could be reinforced (Frank et al. 2015; Liu et al. 2015).

In mammals, perceived temperatures trigger both physiological and behavioral thermoregulatory actions, including thermosensation, hibernation, mating, migration, etc. (Schmidt-Nielsen 1997; Vriens et al. 2014). As for thermosensation, activation of thermoTRPs at the peripheral nerve terminals of DRG and TG neurons send temperature information to the pons, thalamus and other regions of the central nervous system, which in turn elicits various behavioral responses such as thermotaxis and thermal avoidance (Caterina 2007; Vriens et al. 2014). A simplified mammalian thermosensation diagram mediated by the well-studied heat sensor TRPV1 and the cold sensor TRPM8 is outlined in Fig. 2b (Caterina et al. 1997; Dhaka et al. 2008; Julius 2013; Peier et al. 2002).

\section{Temperature and longevity}

In addition to behavior, the sensory environment has a profound impact on many other aspects of life, including aging. For example, studies in C. elegans and Drosophila show that chemosensory neurons regulate lifespan presumably by sensing chemical cues from the environment (Alcedo and Kenyon 2004; Apfeld and Kenyon 1999; Libert et al. 2007; Linford et al. 2011; Ostojic et al. 2014; Waterson et al. 2014). As a sensory cue, temperature influences aging in both poikilotherms and homeotherms. The lifespan of poikilothermic animals, such as worms, flies, and fish, shows an inverse correlation with ambient temperatures; namely, with the exception of extreme temperatures which may threaten survival, animals exhibit a longer lifespan at lower temperatures but a shorter lifespan at warmer temperatures (Conti 2008; Klass 1977; Loeb 1908; Loeb and Northrop 1916). Both body and environmental temperatures regulate the lifespan of homeotherms as well. For example, lowering the core body temperature of mice increases lifespan (Conti et al. 2006). In addition, lower core body temperature is associated with longer lifespan in both mammals and humans (Hauck et al. 2001; Hunter et al. 1999; Roth et al. 2002). In the case of environmental temperature, brief exposure to cool temperatures on daily basis extends the lifespan of rats and protects them from aging-associated diseases (Holloszy and Smith 1986). Furthermore, therapeutic hypothermia has long been used to treat various aging- 
associated cardiovascular arrest, stroke, and brain injury in animal models and humans (Diller and Zhu 2009). All these observations point to a general role of temperature reduction in lifespan extension. However, unlike dietary modulation of longevity which has been extensively characterized, little is known about the mechanisms by which temperature regulates lifespan.

The rate of living theory suggests that the extended and shortened lifespan at low and warm temperatures is attributed to the reduced and increased chemical reaction rates, respectively, and that genetic programs are not involved in this process (Holloszy and Smith 1986; Lints 1989). However, as genetic mechanisms play active roles in all known aging paradigms, one may wonder if temperature modulation of longevity is truly an exception.

\section{A) Temperature modulation of longevity in C. elegans}

Using C. elegans as a model, a recent study has investigated whether genetic pathways actively regulate lifespan at low temperatures (Xiao et al. 2013). This study hypothesized that a thermosensor(s) may detect temperature drop in the environment to initiate longevity signaling, thereby actively promoting lifespan at low temperatures. TRPM8, the best characterized cold sensor in mammals, does not have a homolog in C. elegans. Therefore, the potential role of another cold sensor, TRPA-1, in longevity was interrogated at different temperatures. trpa- 1 mutant worms are short-lived at low temperatures $\left(15^{\circ} \mathrm{C}\right.$ and $\left.20^{\circ} \mathrm{C}\right)$ but not warm temperature $\left(25^{\circ} \mathrm{C}\right)$ compared to wild type worms. Conversely, overexpression of TRPA- 1 extends lifespan at low but not warm temperatures. Thus, TRPA-1 promotes lifespan in a temperature-dependent manner. Given the molecular nature of TRPA- 1 as a thermosensor, these results support the notion that TRPA-1 detects temperature drop in the environment to promote longevity. TRPA-1 does so by recruiting a signaling cascade, including TRPA-1-mediated calcium influx, PKC-2 (a calcium-sensitive protein kinase C), SGK-1 (serum-and glucocorticoid-inducible kinase-1, a DAF-16/FOXO kinase), and the FOXO transcription factor DAF-16, which is a key regulator of lifespan (Fig. 3a) (Xiao et al. 2013). Interestingly, SGK-1 and its upstream mTOR complex 2 (mTORC2) are also reported to modulate $C$. elegans lifespan in a context-dependent fashion (Mizunuma et al. 2014). At low temperatures, SGK-1 promotes lifespan by upregulating DAF-16 (Mizunuma et al. 2014; Xiao et al. 2013), whereas dietary input such as HT115 bacterium can direct SGK-1 to inhibit the Nrf transcription factor SKN-1, another major regulator of lifespan, thereby shortening lifespan (Mizunuma et al. 2014).

Expression of TRPA- 1 in either neurons or the intestine is sufficient to extend lifespan. The nervous system and intestine/fat are the two primary signaling tissues that coordinate bodywide longevity signaling in C. elegans (Kenyon 2010; Libina et al. 2003). Notably, the nervous system and fat tissues also play a critical role in aging in mammals (Timiras 2003). While the underlying cold-sensitive neurons have not yet been identified in C. elegans, intestinal cells appear to directly sense cooling, as acutely isolated intestine responds to temperature decrease. This temperature sensitivity is diminished in trpa-1 mutant worms, supporting that it is mediated by TRPA- 1 . Together, these results argue that low temperature-promoted lifespan extension is not a pure passive thermodynamic phenomenon 
but rather a process that is actively regulated by genes. Nevertheless, it should be noted that trpa-1 mutants still exhibit some temperature sensitivity, suggesting the presence of other cold sensors and perhaps other genetic pathways. Indeed, low temperatures are known to induce the expression of a group of cold-shock proteins (CSPs) across species from bacteria to humans, with many of them belonging to the RNA-binding protein family (Horn et al. 2007). These CSPs could mediate certain protective effects of cooling during neurodegeneration and aging (Peretti et al. 2015). Low temperatures can also stimulate the proteasome degradation pathway in fish, which may contribute to longevity (Lu and Hsu 2015). It is unclear how CSPs and the proteasome pathway are turned on by low temperatures. One possibility is that this could be triggered by cell surface cold receptors in sensory neurons and other thermosensitive tissues. Lastly, the passive thermodynamic mechanism may also contribute to lifespan extension at low temperatures.

C. elegans has a shorter lifespan at high temperatures. The question arises as to whether genetic components are actively involved in shortening lifespan when the environmental temperature increases. Interestingly the opposite has been revealed: genetic components extend lifespan at high temperatures (Jeong et al. 2012; Lee and Kenyon 2009) and this effect is mediated by AFD neuron, a highly versatile heat-sensitive neuron that also regulates thermosensory behaviors. Additionally, AFD neuron mediates a body-wide heatshock cellular response cell-non-autonomously (Prahlad et al. 2008; Sugi et al. 2011). Ablation of AFD or mutations reducing AFD activity further shortens lifespan at high but not low temperatures, demonstrating that AFD neuron antagonizes the detrimental effect of high temperatures on longevity. DAF-9/Cytochrome P450 and the nuclear hormone receptor DAF-12, but not the FOXO transcription factor DAF-16, are required for AFD neuron to promote or maintain normal lifespan at high temperatures (Fig. 3b). Furthermore, HSF-1/ Heat Shock Factor-1 may act independently of AFD neuron and DAF-9/DAF-12 signaling to promote C. elegans lifespan at high temperatures (Fig. 3b) (Jeong et al. 2012; Lee and Kenyon 2009). In addition to AFD, two other thermosensory neurons involved in thermotaxis, AWC and ASI, may also play a role in lifespan regulation. Laser ablation of ASI neuron or a combination of ASI and AWC neurons extends lifespan (Alcedo and Kenyon 2004). As both ASI and AWC are also chemosensory neurons (Bargmann 2006), another possibility is that they regulate longevity through chemosensory signaling. Proper protein folding is critical for cellular physiology (Labbadia and Morimoto 2015).

Temperature has a great impact on protein folding, and various molecular chaperones play essential roles in assisting protein folding (Labbadia and Morimoto 2015). DAF-41/ ZC395.10, the $C$. elegans homolog of p23 chaperone, regulates $C$. elegans longevity in a temperature-dependent manner (Horikawa et al. 2015). daf-41 mutant worms are long-lived at high temperatures (e.g., $25^{\circ} \mathrm{C}$ ) but short-lived at low temperatures (Horikawa et al. 2015). The two opposing longevity phenotypes are mediated by two different sets of transcription factors. It is not clear whether DAF-41 interacts with the TRPA-1- and AFD-dependent pathways. Collectively, these studies clearly demonstrate that genetic pathways play active roles in regulating lifespan at both low and high temperatures.

Although genetic studies described above reveal a critical role of genes in lifespan regulation in response to temperature alternations, the "temperature law" remains: lower 
temperatures extend lifespan while higher temperatures shorten it. This "temperature law", though widely accepted, has not been extensively tested until recently (Zhang et al. 2015). In this study, Zhang et al systematically evaluated the role of temperature on longevity in $C$. elegans, and found that temperature differentially regulates lifespan at different stages of animal life. While exposure to low temperatures at the adult stage prolongs lifespan, low temperature treatment at the larval stage surprisingly reduces lifespan (Fig. 3a) (Zhang et al. 2015). This developmental stage-dependent differential effect of temperature on longevity is mediated by the same thermosensitive TRP channel TRPA-1 that signals to the transcription factor DAF-16/FOXO (Fig. 3a). At the larval stage, TRPA-1 likely acts as a direct coldsensor to shorten lifespan through DAF-16/FOXO, while HSF-1 indirectly responds to heat to extend lifespan (Zhang et al. 2015). At the adult stage, by contrast, TRPA-1 directly detects environmental temperature drop and promotes longevity at low temperatures through DAF-16/FOXO (Fig. 3a). This raises an interesting question: how could DAF-16 produce two opposite lifespan outcomes in larvae and adults? Through microarray analysis and quantitative PCR assays, it was shown that DAF-16 differentially regulates gene expression in larvae and adults in a temperature-dependent manner (Fig. 3a) (Zhang et al. 2015). Such DAF-16-dependent differential regulation of gene expression in larva and adulthood may explain the distinct effects of temperature on longevity at the larval and adult stages. The fact that temperature treatment at the larval stage affects adult lifespan also uncovers an interesting phenomenon: temperature experience in the early life of an animal can induce a long-lasting effect on its late life. Apparently, temperature modulation of longevity in $C$. elegans is much more complex than previously thought and demands further investigations.

\section{B) Temperature modulation of longevity in homeotherms}

The longevity of homeothermic animals is also regulated by temperature, though little is known about the mechanism. Lowering the core body temperature of mice by $0.5^{\circ} \mathrm{C}$ in a transgenic model increases lifespan by $12-20 \%$ (Conti et al. 2006). In addition, mice under DR and those showing hypopituitary and growth hormone-resistance all display a lower core body temperature and are long-lived (Hauck et al. 2001; Hunter et al. 1999). Notably, a reduced core body temperature is also associated with longer human lifespan as shown in data from the Baltimore Longitudinal Study of Aging (BLSA) (Roth et al. 2002). Therefore, both body and environmental temperatures modulate longevity in homeotherms. However, the underlying genetic mechanisms remain largely unexplored.

As many thermoTRPs are expressed in both the peripheral and central nervous systems, they may play important roles in transforming temperature information into longevity output. Knockout mice lacking TRPV1, a heat and capsaicin receptor, are long-lived, demonstrating that mouse TRPV1 shortens lifespan (Riera et al. 2014). A similar phenomenon was also observed in mutant worms deficient in the TRPV channels OSM-9 and OCR-2 (Lee and Ashrafi 2008; Riera et al. 2014). TRPV1 in DRG neurons triggers intracellular calcium signaling and modulates longevity by activating CRTC, a CREB-regulated transcriptional coactivator (Riera et al. 2014). Together with CREB, CRTC promotes the transcription and release of the neuropeptide calcitonin gene-related peptide (CGRP) from DRG sensory endings, which in turn inhibits insulin release from pancreatic $\beta$ cells, leading to aging- 
associated metabolic decline (Riera et al. 2014). As TRPV1 is a heat-sensitive channel enriched in sensory neurons, this raises the interesting possibility that TRPV1 or other thermoTRPs may act as a peripheral thermosensor to detect temperature increase in the environment to shorten lifespan in mice.

Fat cells are known to be critical in mammalian insulin signaling, metabolism and aging (Picard and Guarente 2005; Tchkonia et al. 2010). These cells respond to ambient temperature changes through sympathetic pathways. Specifically, upon low temperature stimulation, cutaneous thermoreceptors (e.g. thermosensitive TRPs) located in primary sensory neurons and skin tissues deliver the temperature information to the preoptic area (POA) and hypothalamus through the spinal and trigeminal dorsal horn. At POA and hypothalamus, the temperature signals are integrated and then transduced to fat cells and other tissues through sympathetic output (Morrison et al. 2012). Indeed, at low temperatures, fat cells exhibit an enhanced expression of multiple thermogenic genes, including uncoupling protein 1 (UCP1), peroxisome proliferator-activated receptor gamma coactivator 1-alpha (PGC-1a), and type II iodothyronine deiodinase (Dio2) (Rosen and Spiegelman 2014). Interestingly, upregulated UCP1 and PGC1a activities are both linked to improved healthspan and extended lifespan in transgenic mouse studies (Gates et al. 2007; Wenz et al. 2009), implying a potential mechanism for promoting longevity at low temperatures in homeotherms. In this regard, it is possible that fat tissues may act downstream of the nervous system to mediate the effect of temperature on longevity in homeotherms. Nevertheless, these are circumstantial evidence at best, and future studies are needed to directly explore the mechanisms underlying temperature modulation of longevity in homeotherms.

\section{Concluding remarks}

Temperature affects nearly every aspect of animal life. Throughout evolution, animals have evolved various mechanisms to respond and adapt to temperature fluctuations in the environment. Remarkably, a subset of thermoTRP channels play evolutionarily conserved roles in thermosensation. In this review, we mainly highlighted our current understanding of molecular thermosensors in model organisms such as C. elegans, Drosophila, and mice. Activation of these thermoTRPs and other molecular thermosensors elicit complex behavioral responses in a context-dependent manner. It is notable that thermoTRP knockout animals still display temperature sensitivity, indicating that other molecular thermosensors are probably present. Further investigations are required to identify and characterize these novel temperature sensors.

In addition to behavior, ambient temperature modulates aging. Both poikilotherms and homeotherms live longer at lower temperatures, and vice versa. Though the rate-of-living mechanism may still contribute, studies in C. elegans demonstrate that genetic programs play important roles in mediating the effect of temperature on longevity. Temperature modulation of longevity is apparently more complex than previously thought, as recent work shows that temperature differentially regulates lifespan in C. elegans at different stages of life. As both neurons and intestinal cells are involved in regulating this process, it is 
interesting to investigate the mechanisms by which neurons communicate with intestinal cells. Moreover, how temperature signals are transmitted from neurons and the intestine to other tissues remains enigmatic.

Body temperature affects lifespan in homeothermic animals; however, the underlying mechanisms remain largely unexplored. Lower body temperature is also linked to longer life expectancy in humans. While lower environmental temperatures extend lifespan in rodents, it is unclear whether this phenomenon can be extended to humans. Similar to C. elegans, certain mammalian tissues are temperature sensitive. These tissues may act as cellular thermosensors mediating the effect of temperature on longevity in mammals. Future studies are needed to verify whether these thermosensitive tissues modulate longevity, identify the thermosensors in these tissues, and investigate how longevity signals are transmitted from thermosensitive tissues to other tissues in mammals. As the aging mechanisms tend to be evolutionarily conserved, work in C. elegans and other simple model organisms will continue to provide novel insights into how temperature modulates longevity in mammals, including humans.

\section{Acknowledgments}

We thank Adam Iliff and other lab members for comments. R.X. is supported by an NIA T32 Training Grant (AG000114). Lab research is supported by grants from the NIH (X.Z.S.X) and the NSFC (31130028, 31225011, and 31420103909 to J.L.), the Program of Introducing Talents of Discipline to Universities from the Ministry of Education of China (B08029 to J.L.), and the Ministry of Science and Technology of China (2012CB51800 to J.L.).

\section{References}

Alcedo J, Kenyon C. Regulation of C. elegans longevity by specific gustatory and olfactory neurons. Neuron. 2004; 41:45-55. [PubMed: 14715134]

Apfeld J, Kenyon C. Regulation of lifespan by sensory perception in Caenorhabditis elegans. Nature. 1999; 402:804-809. [PubMed: 10617200]

Bargmann CI. Chemosensation in C. elegans. WormBook. 2006:1-29. [PubMed: 18050433]

Bishop NA, Guarente L. Genetic links between diet and lifespan: shared mechanisms from yeast to humans. Nat Rev Genet. 2007; 8:835-844. [PubMed: 17909538]

Bounoutas A, Chalfie M. Touch sensitivity in Caenorhabditis elegans. Pflugers Arch. 2007; 454:691702. [PubMed: 17285303]

Cabanac M. Temperature regulation. Annu Rev Physiol. 1975; 37:415-439. [PubMed: 123725]

Caterina MJ. Transient receptor potential ion channels as participants in thermosensation and thermoregulation. Am J Physiol Regul Integr Comp Physiol. 2007; 292:R64-76. [PubMed: 16973931]

Caterina MJ. TRP Channel Cannabinoid Receptors in Skin Sensation, Homeostasis, and Inflammation. ACS Chem Neurosci. 201410.1021/cn5000919

Caterina MJ, Schumacher MA, Tominaga M, Rosen TA, Levine JD, Julius D. The capsaicin receptor: a heat-activated ion channel in the pain pathway. Nature. 1997; 389:816-824. [PubMed: 9349813]

Chatzigeorgiou M, Schafer WR. Lateral Facilitation between Primary Mechanosensory Neurons Controls Nose Touch Perception in C. elegans. Neuron. 2011; 70:299-309. [PubMed: 21521615]

Chatzigeorgiou M, et al. Specific roles for DEG/ENaC and TRP channels in touch and thermosensation in C. elegans nociceptors. Nat Neurosci. 2010; 13:861-868. [PubMed: 20512132]

Chowdhury S, Jarecki BW, Chanda B. A molecular framework for temperature-dependent gating of ion channels. Cell. 2014; 158:1148-1158. [PubMed: 25156949] 
Clapham DE, Miller C. A thermodynamic framework for understanding temperature sensing by transient receptor potential (TRP) channels. Proc Natl Acad Sci U S A. 2011; 108:19492-19497. [PubMed: 22109551]

Clarke A, Portner HO. Temperature, metabolic power and the evolution of endothermy. Biol Rev Camb Philos Soc. 2010; 85:703-727. [PubMed: 20105154]

Conti B. Considerations on temperature, longevity and aging. Cell Mol Life Sci. 2008; 65:1626-1630. [PubMed: 18425417]

Conti B, et al. Transgenic mice with a reduced core body temperature have an increased life span. Science. 2006; 314:825-828. [PubMed: 17082459]

Dhaka A, Earley TJ, Watson J, Patapoutian A. Visualizing cold spots: TRPM8-expressing sensory neurons and their projections. J Neurosci. 2008; 28:566-575. [PubMed: 18199758]

Dhaka A, Viswanath V, Patapoutian A. Trp ion channels and temperature sensation. Annu Rev Neurosci. 2006; 29:135-161. [PubMed: 16776582]

Diller KR, Zhu L. Hypothermia therapy for brain injury. Annu Rev Biomed Eng. 2009; 11:135-162. [PubMed: 19400711]

Finch CE, Ruvkun G. The genetics of aging. Annu Rev Genomics Hum Genet. 2001; 2:435-462. [PubMed: 11701657]

Fowler MA, Montell C. Drosophila TRP channels and animal behavior. Life Sci. 2013; 92:394-403. [PubMed: 22877650]

Frank DD, Jouandet GC, Kearney PJ, Macpherson LJ, Gallio M. Temperature representation in the Drosophila brain. Nature. 2015; 519:358-361. [PubMed: 25739506]

Gallio M, Ofstad TA, Macpherson LJ, Wang JW, Zuker CS. The coding of temperature in the Drosophila brain. Cell. 2011; 144:614-624. [PubMed: 21335241]

Garrity PA, Goodman MB, Samuel AD, Sengupta P. Running hot and cold: behavioral strategies, neural circuits, and the molecular machinery for thermotaxis in C. elegans and Drosophila. Genes Dev. 2010; 24:2365-2382. [PubMed: 21041406]

Gates AC, et al. Respiratory uncoupling in skeletal muscle delays death and diminishes age-related disease. Cell Metab. 2007; 6:497-505. [PubMed: 18054318]

Gems D, Partridge L. Genetics of longevity in model organisms: debates and paradigm shifts. Annual review of physiology. 2013; 75:621-644.

Glauser DA, et al. Heat avoidance is regulated by transient receptor potential (TRP) channels and a neuropeptide signaling pathway in Caenorhabditis elegans. Genetics. 2011; 188:91-103. [PubMed: 21368276]

Gracheva EO, et al. Ganglion-specific splicing of TRPV1 underlies infrared sensation in vampire bats. Nature. 2011; 476:88-91. [PubMed: 21814281]

Gracheva EO, et al. Molecular basis of infrared detection by snakes. Nature. 2010; 464:1006-1011. [PubMed: 20228791]

Gurland BJ, Page WF, Plassman BL. A twin study of the genetic contribution to age-related functional impairment. J Gerontol A Biol Sci Med Sci. 2004; 59:859-863. [PubMed: 15345739]

Hamada FN, Rosenzweig M, Kang K, Pulver SR, Ghezzi A, Jegla TJ, Garrity PA. An internal thermal sensor controlling temperature preference in Drosophila. Nature. 2008; 454:217-220. [PubMed: 18548007]

Hauck SJ, Hunter WS, Danilovich N, Kopchick JJ, Bartke A. Reduced levels of thyroid hormones, insulin, and glucose, and lower body core temperature in the growth hormone receptor/binding protein knockout mouse. Exp Biol Med (Maywood). 2001; 226:552-558. [PubMed: 11395925]

Hedgecock EM, Russell RL. Normal and mutant thermotaxis in the nematode Caenorhabditis elegans. Proc Natl Acad Sci U S A. 1975; 72:4061-4065. [PubMed: 1060088]

Herskind AM, McGue M, Holm NV, Sorensen TI, Harvald B, Vaupel JW. The heritability of human longevity: a population-based study of 2872 Danish twin pairs born 1870-1900. Hum Genet. 1996; 97:319-323. [PubMed: 8786073]

Hille, B. Ion channels of excitable membranes. 3. Sinauer; Sunderland, Mass: 2001. 
Holloszy JO, Smith EK. Longevity of cold-exposed rats: a reevaluation of the "rate-of-living theory". J Appl Physiol (1985). 1986; 61:1656-1660. [PubMed: 3781978]

Horikawa M, Sural S, Hsu AL, Antebi A. Co-chaperone p23 Regulates C. elegans Lifespan in Response to Temperature. PLoS Genet. 2015; 11:e1005023. [PubMed: 25830239]

Horn G, Hofweber R, Kremer W, Kalbitzer HR. Structure and function of bacterial cold shock proteins. Cell Mol Life Sci. 2007; 64:1457-1470. [PubMed: 17437059]

Hunter WS, Croson WB, Bartke A, Gentry MV, Meliska CJ. Low body temperature in long-lived Ames dwarf mice at rest and during stress. Physiol Behav. 1999; 67:433-437. [PubMed: 10497963]

Jabba S, et al. Directionality of temperature activation in mouse TRPA1 ion channel can be inverted by single-point mutations in ankyrin repeat six. Neuron. 2014; 82:1017-1031. [PubMed: 24814535]

Jeong DE, Artan M, Seo K, Lee SJ. Regulation of lifespan by chemosensory and thermosensory systems: findings in invertebrates and their implications in mammalian aging. Front Genet. 2012; 3:218. [PubMed: 23087711]

Jordt SE, McKemy DD, Julius D. Lessons from peppers and peppermint: the molecular logic of thermosensation. Curr Opin Neurobiol. 2003; 13:487-492. [PubMed: 12965298]

Julius D. TRP channels and pain. Annu Rev Cell Dev Biol. 2013; 29:355-384. [PubMed: 24099085]

Kahn-Kirby AH, Bargmann CI. TRP channels in C. elegans. Annu Rev Physiol. 2006; 68:719-736. [PubMed: 16460289]

Kang K, et al. Modulation of TRPA1 thermal sensitivity enables sensory discrimination in Drosophila. Nature. 2012; 481:76-80. [PubMed: 22139422]

Kenyon CJ. The genetics of ageing. Nature. 2010; 464:504-512. [PubMed: 20336132]

Klass MR. Aging in the nematode Caenorhabditis elegans: major biological and environmental factors influencing life span. Mech Ageing Dev. 1977; 6:413-429. [PubMed: 926867]

Kwon Y, Shen WL, Shim HS, Montell C. Fine thermotactic discrimination between the optimal and slightly cooler temperatures via a TRPV channel in chordotonal neurons. J Neurosci. 2010; 30:10465-10471. [PubMed: 20685989]

Kwon Y, Shim HS, Wang X, Montell C. Control of thermotactic behavior via coupling of a TRP channel to a phospholipase C signaling cascade. Nat Neurosci. 2008; 11:871-873. [PubMed: 18660806]

Labbadia J, Morimoto RI. The Biology of Proteostasis in Aging and Disease. Annu Rev Biochem. 201510.1146/annurev-biochem-060614-033955

Lee BH, Ashrafi K. A TRPV channel modulates C. elegans neurosecretion, larval starvation survival, and adult lifespan. PLoS Genet. 2008; 4:e1000213. [PubMed: 18846209]

Lee SJ, Kenyon C. Regulation of the longevity response to temperature by thermosensory neurons in Caenorhabditis elegans. Curr Biol. 2009; 19:715-722. [PubMed: 19375320]

Lee $\mathrm{Y}$, et al. Pyrexia is a new thermal transient receptor potential channel endowing tolerance to high temperatures in Drosophila melanogaster. Nat Genet. 2005; 37:305-310. [PubMed: 15731759]

Lee Y, Montell C. Drosophila TRPA1 functions in temperature control of circadian rhythm in pacemaker neurons. J Neurosci. 2013; 33:6716-6725. [PubMed: 23595730]

Li W, Feng Z, Sternberg PW, Xu XZS. A C. elegans stretch receptor neuron revealed by a mechanosensitive TRP channel homologue. Nature. 2006; 440:684-687. [PubMed: 16572173]

Libert S, Zwiener J, Chu X, Vanvoorhies W, Roman G, Pletcher SD. Regulation of Drosophila life span by olfaction and food-derived odors. Science. 2007; 315:1133-1137. [PubMed: 17272684]

Libina N, Berman JR, Kenyon C. Tissue-specific activities of C. elegans DAF-16 in the regulation of lifespan. Cell. 2003; 115:489-502. [PubMed: 14622602]

Linford NJ, Kuo TH, Chan TP, Pletcher SD. Sensory perception and aging in model systems: from the outside in. Annual review of cell and developmental biology. 2011; 27:759-785.

Lints FA. The rate of living theory revisited. Gerontology. 1989; 35:36-57. [PubMed: 2656413]

Liu S, Schulze E, Baumeister R. Temperature- and touch-sensitive neurons couple CNG and TRPV channel activities to control heat avoidance in Caenorhabditis elegans. PLoS One. 2012; 7:e32360. [PubMed: 22448218] 
Liu WW, Mazor O, Wilson RI. Thermosensory processing in the Drosophila brain. Nature. 2015; 519:353-357. [PubMed: 25739502]

Loeb J. Ueber den Temperaturkoeffizienten für die Lebensdauer kaltblütiger Thiere und über die Ursache des natürlichen Todes. Pflüger Arch. 1908; 124:411-426.

Loeb J, Northrop JH. Is there a temperature coefficient for the duration of life? P Natl Acad Sci USA. $1916 ; 2: 456-457$.

Lotshaw DP. Biophysical, pharmacological, and functional characteristics of cloned and native mammalian two-pore domain K+ channels. Cell Biochem Biophys. 2007; 47:209-256. [PubMed: 17652773]

Lu CY, Hsu CY. Ambient temperature reduction extends lifespan via activating cellular degradation activity in an annual fish (Nothobranchius rachovii). Age (Dordr). 2015; 37:9775.

Luo DG, Yue WW, Ala-Laurila P, Yau KW. Activation of visual pigments by light and heat. Science. 2011; 332:1307-1312. [PubMed: 21659602]

Luo L, et al. Bidirectional thermotaxis in Caenorhabditis elegans is mediated by distinct sensorimotor strategies driven by the AFD thermosensory neurons. Proc Natl Acad Sci U S A. 2014; 111:27762781. [PubMed: 24550307]

Mair W, Dillin A. Aging and survival: the genetics of life span extension by dietary restriction. Annu Rev Biochem. 2008; 77:727-754. [PubMed: 18373439]

McGhee JD. The C. elegans intestine. WormBook. 2007:1-36.10.1895/wormbook.1.133.1 [PubMed: 18050495]

McKemy DD. Temperature sensing across species. Pflugers Arch. 2007; 454:777-791. [PubMed: 17219191]

Mizunuma M, Neumann-Haefelin E, Moroz N, Li Y, Blackwell TK. mTORC2-SGK-1 acts in two environmentally responsive pathways with opposing effects on longevity. Aging Cell. 2014; 13:869-878. [PubMed: 25040785]

Mohammadi A, Byrne Rodgers J, Kotera I, Ryu WS. Behavioral response of Caenorhabditis elegans to localized thermal stimuli. BMC Neurosci. 2013; 14:66. [PubMed: 23822173]

Montell C, Rubin GM. Molecular characterization of the Drosophila trp locus: a putative integral membrane protein required for phototransduction. Neuron. 1989; 2:1313-1323. [PubMed: 2516726]

Mori I, Sasakura H, Kuhara A. Worm thermotaxis: a model system for analyzing thermosensation and neural plasticity. Curr Opin Neurobiol. 2007; 17:712-719. [PubMed: 18242074]

Morrison SF, Madden CJ, Tupone D. Central control of brown adipose tissue thermogenesis. Front Endocrinol (Lausanne). 2012; 3

$\mathrm{Ni}$ L, et al. A gustatory receptor paralogue controls rapid warmth avoidance in Drosophila. Nature. 2013; 500:580-584. [PubMed: 23925112]

Ostojic I, Boll W, Waterson MJ, Chan T, Chandra R, Pletcher SD, Alcedo J. Positive and negative gustatory inputs affect Drosophila lifespan partly in parallel to dFOXO signaling. Proc Natl Acad Sci U S A. 2014; 111:8143-8148. [PubMed: 24847072]

Peier AM, et al. A TRP channel that senses cold stimuli and menthol. Cell. 2002; 108:705-715. [PubMed: 11893340]

Peretti D, et al. RBM3 mediates structural plasticity and protective effects of cooling in neurodegeneration. Nature. 2015; 518:236-239. [PubMed: 25607368]

Picard F, Guarente L. Molecular links between aging and adipose tissue. Int J Obes (Lond). 2005; 29(Suppl 1):S36-39. [PubMed: 15711582]

Prahlad V, Cornelius T, Morimoto RI. Regulation of the cellular heat shock response in Caenorhabditis elegans by thermosensory neurons. Science. 2008; 320:811-814. [PubMed: 18467592]

Ramot D, MacInnis BL, Goodman MB. Bidirectional temperature-sensing by a single thermosensory neuron in C. elegans. Nat Neurosci. 2008; 11:908-915. [PubMed: 18660808]

Ramsey IS, Delling M, Clapham DE. An introduction to TRP channels. Annu Rev Physiol. 2006; 68:619-647. [PubMed: 16460286] 
Riera CE, et al. TRPV1 pain receptors regulate longevity and metabolism by neuropeptide signaling. Cell. 2014; 157:1023-1036. [PubMed: 24855942]

Rosen ED, Spiegelman BM. What we talk about when we talk about fat. Cell. 2014; 156:20-44. [PubMed: 24439368]

Rosenzweig M, Brennan KM, Tayler TD, Phelps PO, Patapoutian A, Garrity PA. The Drosophila ortholog of vertebrate TRPA1 regulates thermotaxis. Genes Dev. 2005; 19:419-424. [PubMed: 15681611]

Rosenzweig M, Kang K, Garrity PA. Distinct TRP channels are required for warm and cool avoidance in Drosophila melanogaster. Proc Natl Acad Sci U S A. 2008; 105:14668-14673. [PubMed: 18787131]

Roth GS, et al. Biomarkers of caloric restriction may predict longevity in humans. Science. 2002; 297:811. [PubMed: 12161648]

Saito S, Fukuta N, Shingai R, Tominaga M. Evolution of vertebrate transient receptor potential vanilloid 3 channels: opposite temperature sensitivity between mammals and western clawed frogs. PLoS Genet. 2011; 7:e1002041. [PubMed: 21490957]

Schmidt-Nielsen, K. Animal physiology : adaptation and environment. 5. Cambridge University Press; Cambridge, U.K.; New York: 1997.

Shen WL, Kwon Y, Adegbola AA, Luo J, Chess A, Montell C. Function of rhodopsin in temperature discrimination in Drosophila. Science. 2011; 331:1333-1336. [PubMed: 21393546]

Story GM, et al. ANKTM1, a TRP-like channel expressed in nociceptive neurons, is activated by cold temperatures. Cell. 2003; 112:819-829. [PubMed: 12654248]

Sugi T, Nishida Y, Mori I. Regulation of behavioral plasticity by systemic temperature signaling in Caenorhabditis elegans. Nat Neurosci. 2011; 14:984-992. [PubMed: 21706021]

Tchkonia T, et al. Fat tissue, aging, and cellular senescence Aging. Cell. 2010; 9:667-684.

Timiras, PS. Physiological basis of aging and geriatrics. 3. CRC Press; Boca Raton, Fla: 2003.

Tracey WD Jr, Wilson RI, Laurent G, Benzer S. painless, a Drosophila gene essential for nociception. Cell. 2003; 113:261-273. [PubMed: 12705873]

Venkatachalam K, Montell C. TRP channels. Annu Rev Biochem. 2007; 76:387-417. [PubMed: 17579562]

Viswanath V, et al. Opposite thermosensor in fruitfly and mouse. Nature. 2003; 423:822-823. [PubMed: 12815418]

Vriens J, Nilius B, Voets T. Peripheral thermosensation in mammals. Nat Rev Neurosci. 2014; 15:573-589. [PubMed: 25053448]

Ward A, Liu J, Feng Z, Xu XZ. Light-sensitive neurons and channels mediate phototaxis in C. elegans. Nat Neurosci. 2008; 11:916-922. [PubMed: 18604203]

Waterson MJ, Chung BY, Harvanek ZM, Ostojic I, Alcedo J, Pletcher SD. Water sensor ppk28 modulates Drosophila lifespan and physiology through AKH signaling. Proc Natl Acad Sci U S A. 2014; 111:8137-8142. [PubMed: 24821805]

Wenz T, Rossi SG, Rotundo RL, Spiegelman BM, Moraes CT. Increased muscle PGC-1alpha expression protects from sarcopenia and metabolic disease during aging. Proc Natl Acad Sci U S A. 2009; 106:20405-20410. [PubMed: 19918075]

White JG, Southgate E, Thomson JN, Brenner S. The structure of the nervous system of the nematode Caenorhabditis elegans. Philos Trans R Soc Lond B Biol Sci. 1986; 314:1-340. [PubMed: 22462104]

Wittenburg N, Baumeister R. Thermal avoidance in Caenorhabditis elegans: an approach to the study of nociception. Proc Natl Acad Sci U S A. 1999; 96:10477-10482. [PubMed: 10468634]

Xiao R, Xu XZS. Function and regulation of TRP family channels in C. elegans. Pflugers Arch. 2009; 458:851-860. [PubMed: 19421772]

Xiao R, Zhang B, Dong Y, Gong J, Xu T, Liu J, Xu XZ. A genetic program promotes C. elegans longevity at cold temperatures via a thermosensitive TRP channel. Cell. 2013; 152:806-817. [PubMed: 23415228] 
Yang F, Zheng J. High temperature sensitivity is intrinsic to voltage-gated potassium channels. Elife. 2014; 3:e03255.10.7554/eLife.03255 [PubMed: 25030910]

Ye L, et al. Fat cells directly sense temperature to activate thermogenesis. Proc Natl Acad Sci U S A. 2013; 110:12480-12485. [PubMed: 23818608]

Zhang B, Xiao R, Ronan EA, He Y, Hsu AL, Liu J, Xu XZ. Environmental Temperature Differentially Modulates C. elegans Longevity through a Thermosensitive TRP Channel. Cell Rep. 2015

Zhong L, et al. Thermosensory and nonthermosensory isoforms of Drosophila melanogaster TRPA1 reveal heat-sensor domains of a thermoTRP Channel. Cell Rep. 2012; 1:43-55. [PubMed: 22347718]

\section{List of abbreviations}

\begin{tabular}{|c|c|}
\hline AC neurons & Anterior cell neurons \\
\hline BLSA & Baltimore Longitudinal Study of Aging \\
\hline CGRP & Calcitonin gene-related peptide \\
\hline CSPs & Cold-shock proteins \\
\hline Dio2 & Type II iodothyronine deiodinase \\
\hline DR & Dietary restriction \\
\hline DRG & Dorsal root ganglia \\
\hline HSF-1 & Heat Shock Factor-1 \\
\hline IGF1 & Insulin-like growth factor 1 \\
\hline IIS & Insulin and insulin-like growth factor 1 signaling \\
\hline MD neurons & Multidendritic neurons \\
\hline mTOR & Mechanistic target of rapamycin \\
\hline mTORC2 & mTOR complex 2 \\
\hline PGC-1a & Peroxisome proliferator-activated receptor gamma coactivator 1-alpha \\
\hline PKC-2 & Protein kinase $\mathrm{C}-2$ \\
\hline POA & Preoptic area \\
\hline SGK-1 & Serum-and glucocorticoid-inducible kinase- 1 \\
\hline TG & Trigeminal ganglia \\
\hline TRP & Transient receptor potential \\
\hline TRPC & TRP-Canonical \\
\hline TRPV & TRP-Vanilloid \\
\hline TRPM & TRP-Melastatin \\
\hline TRPN & TRP-NompC \\
\hline TRPA & TRP-Ankyrin \\
\hline TRPP & TRP-Polycystin \\
\hline
\end{tabular}


TRPML

UCP1
TRP-MucoLipin

Uncoupling protein 1 


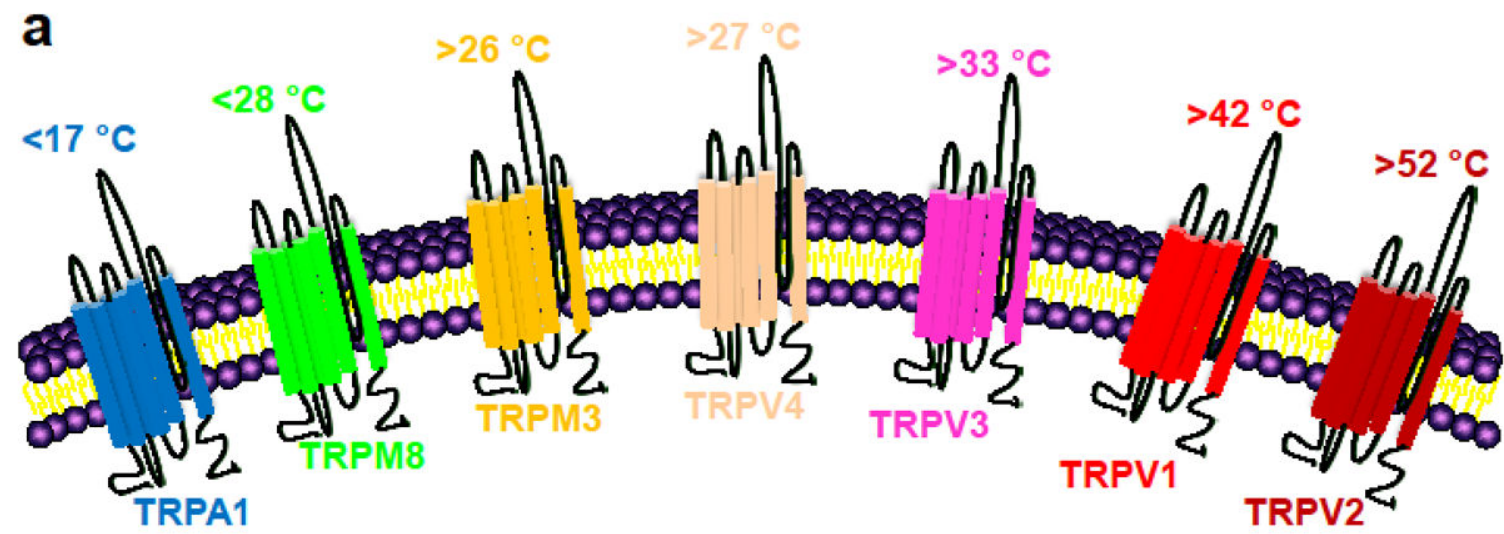

b

\begin{tabular}{lll}
\multicolumn{3}{c}{ Drosophila molecular thermosensors } \\
\hline Channel & $\begin{array}{l}\text { T } \\
\text { (activation) }\end{array}$ & Physiological functions \\
\hline TRPA1 & $\sim 26^{\circ} \mathrm{C}$ & $\begin{array}{l}\text { Sensing warm temperature, } \\
\text { avoiding aversive odorants } \\
\text { and short wavelength light }\end{array}$ \\
Painless & $\sim 39^{\circ} \mathrm{C}$ & $\begin{array}{l}\text { Sensing noxious heat and } \\
\text { gravity } \\
\text { Sensing noxious heat and } \\
\text { gravity }\end{array}$ \\
\hline
\end{tabular}

C

C. elegans molecular thermosensors

\begin{tabular}{lll}
\hline Channel & $\begin{array}{l}\text { T } \\
\text { (activation) }\end{array}$ & Physiological functions \\
\hline TRPA-1 & $\sim 20^{\circ} \mathrm{C}$ & $\begin{array}{l}\text { Direct sensor of cold in } \\
\text { neurons and intestine; } \\
\text { modulation of } \\
\text { mechanosensation. }\end{array}$ \\
& & $\begin{array}{l}\text { Directly or indirectly } \\
\text { modulating noxious heat } \\
\text { avoidance }\end{array}$ \\
OSM-9 & $\sim 35^{\circ} \mathrm{C}$ & \\
\hline
\end{tabular}

Figure 1. Molecular thermosensors in mammals, Drosophila, and C. elegans a, mammalian thermoTRPs activated by cold (TRPM8 and TRPA1) and heat (TRPV1-V4 and TRPM3) are depicted, with their approximate temperature activation threshold highlighted. b, Drosophila thermosensors and their physiological functions. c, C. elegans thermosensors and their physiological functions. 
a

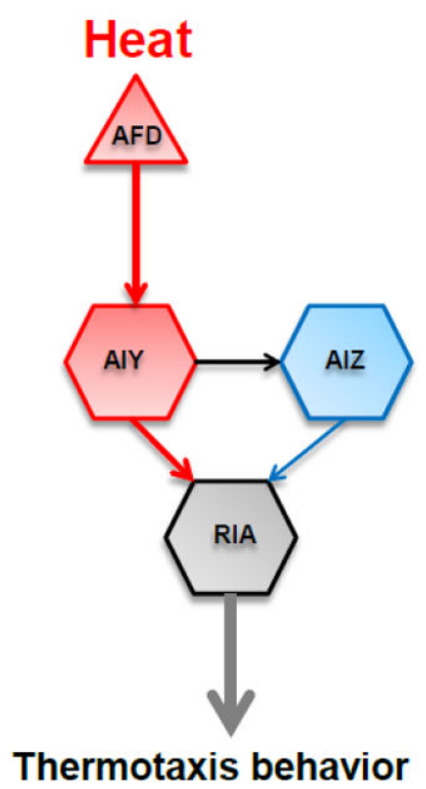

b

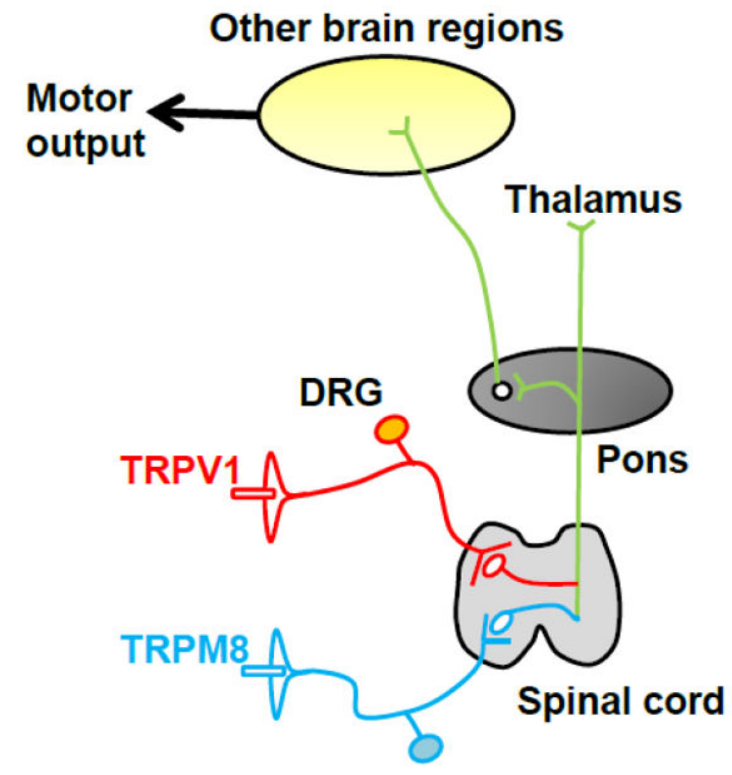

Figure 2. Neural circuits underlying temperature-regulated behaviors a, neural circuitry of thermotaxis behavior in C. elegans. b, neural circuitry of thermosensation in mammals. 


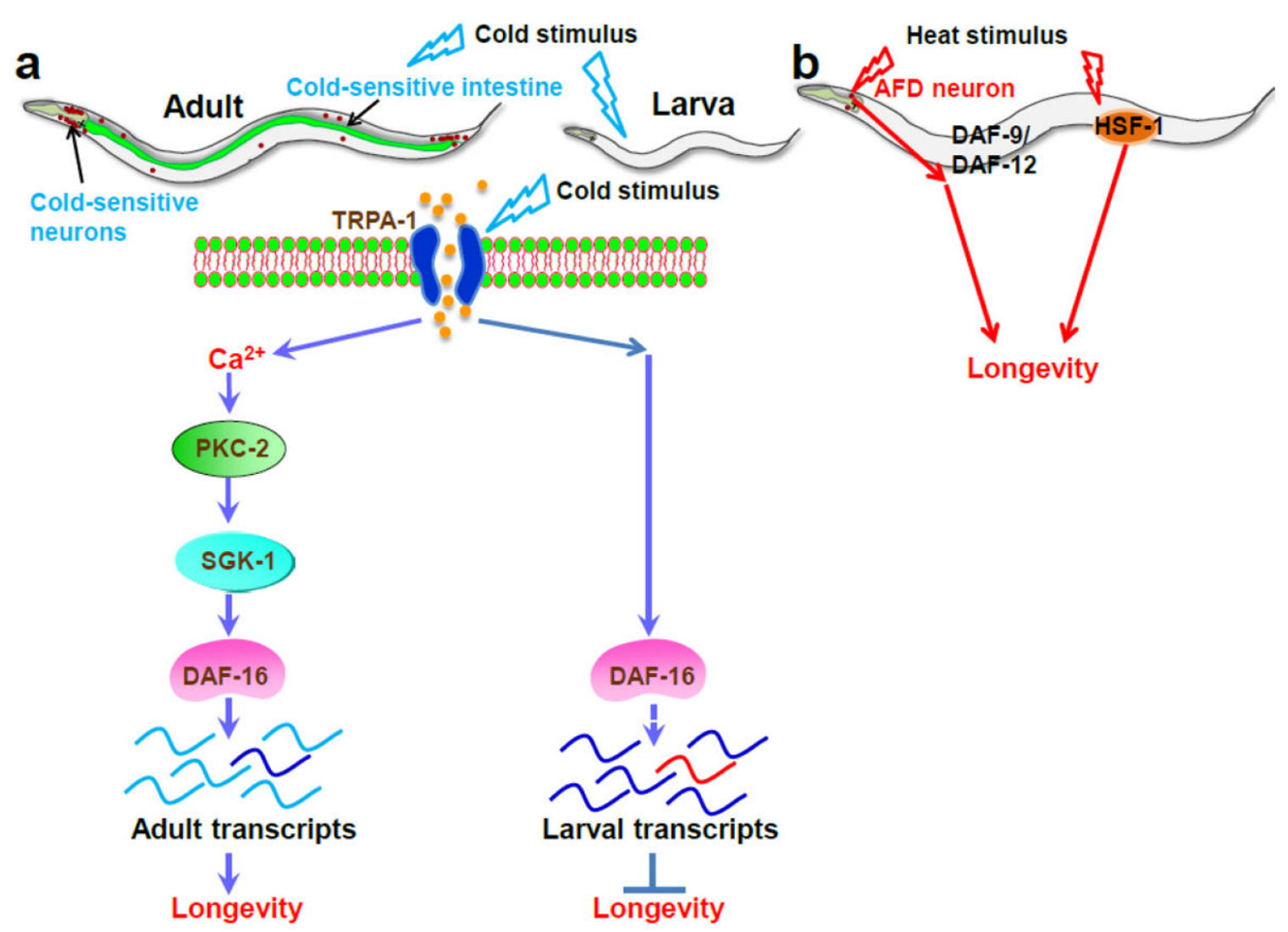

Figure 3. Genetic mechanisms underlying temperature modulation of longevity in C. elegans a, in adult worms, a TRPA-1-initiated genetic program promotes lifespan at low temperatures through low temperature-induced calcium influx, PKC-2, SGK-1, and DAF-16. By contrast, TRPA-1 at the larval stage shortens adult lifespan through DAF-16 by differentially regulating target gene expression patterns. $\mathbf{b}$, the AFD neuron detects heat stimulus and transmits the temperature signal to DAF-9/DAF-12 in a cell non-autonomous manner, thereby counteracting the life-shortening effect of high temperatures. HSF-1 may sense heat, albeit indirectly, through heat-shock signaling to extend lifespan in both adult and larva. 